\title{
All-Versus-Nothing Violation of Local Realism for Two Entangled Photons
}

\author{
Zeng-Bing Chen, ${ }^{1}$ Jian-Wei Pan, ${ }^{2}$ Yong-De Zhang, ${ }^{1}$ Caslav Brukner, ${ }^{2}$ and Anton Zeilinger ${ }^{2}$ \\ ${ }^{1}$ Department of Modern Physics, University of Science and Technology of China, Hefei, Anhui 230027, China \\ ${ }^{2}$ Institut für Experimentalphysik, Universität Wien, Boltzmanngasse 5, 1090 Wien, Austria
}

\begin{abstract}
It is shown that the Greenberger-Horne-Zeilinger theorem can be generalized to the case with only two entangled particles. The reasoning makes use of two photons which are maximally entangled both in polarization and in spatial degrees of freedom. In contrast to Cabello's argument of "all versus nothing" nonlocality with four photons [Phys. Rev. Lett. 87, 010403 (2001)], our proposal to test the theorem can be implemented with linear optics and thus is well within the reach of current experimental technology.
\end{abstract}

PACS numbers: 03.65.Ud, 03.65.Ta, 03.67.-a, 42.50.-p

Bell's theorem [1], which is derived from Einstein, Podolsky, and Rosen's (EPR's) notion of local realism [2], represents the most radical departure of quantum mechanics (QM) from one's classical intuitions. On the one hand, Bell's inequalities (BI) state that certain statistical correlations predicted by QM for measurements on two-particle ensembles cannot be understood within a realistic picture based on local properties of each individual particle. On the other hand, an unstatisfactory feature in the derivation of $\mathrm{BI}$ is that such a local realistic and thus classical picture can explain perfect correlations and is only in conflict with statistical prediction of the theory.

Strikingly, "Bell's theorem without inequalities" has been demonstrated for multiparticle Greenberger-HorneZeilinger (GHZ) states [3, 4, 5], where the contradiction between QM and local realistic theories arises even for definite predictions. The quantum nonlocality can thus, in principle, be manifest in a single run of a certain measurement. This is known as the "all versus nothing" proof of Bell's theorem. In addition, the GHZ contradiction applies for all (100\%) multiparticle systems that are in the same GHZ state. In the sense that it is for definite predictions and for all systems the GHZ theorem represents the strongest conflict between $\mathrm{QM}$ and local realism. However, the GHZ reasoning requires at least three particles and, consequently, three space-like separated regions (observers). This can be seen as a sort of three-particle quantum nonlocality, which differs from the two-particle quantum nonlocality as implied in usual BI.

Then Hardy's argument of "quantum nonlocality without inequalities" for nonmaximally entangled biparticle states [6] came as a surprise. Now it is known as "the best version of Bell's theorem" 7] for two-dimensional two-particle systems. However, compared to the GHZ case, in Hardy's proof only a fraction $(\lesssim 9 \%)$ of the photon pairs shows a contradiction with local realism. Most recently, another way to reveal sharper violations of local realism for two-particle entangled states in higherdimensional Hilbert spaces was found [8, 9]. For the two-particle entangled states of high-dimensionality, the violation of local realism has more resistance to noise, but is still statistical. Motivated by Hardy and the highdimensional versions of Bell's theorem, one may ask: Can the conflict between QM and local realism arise even for the definite predictions and for all (100\%) of the photon pairs in the same entangled state?

In this Letter we answer the question affirmatively by demonstrating an all-versus-nothing nonlocality for two photons which are maximally entangled both in polarization and in spatial (path) degrees of freedom. Such a "double entanglement" plays a crucial role in our demonstration. From a formal aspect, our demonstration is a further development of Cabello's [10, 11] elegant proof of quantum nonlocality without inequalities for two observers who possess two pairs of maximally entangled qubits, i.e., four two-level particles. Lvovsky demonstrated that the nonlinear optics at a single-photon level is required for a demonstration of Cabello's quantum nonlocality without implicit assumption of noncontextuality 12. Unfortunately, such an experimental test of Cabello's quantum nonlocality is beyond the present level of quantum optical technology. By contrast, the experiment proposed here has two advantanges over Cabello's proposal. First, the observers need to possess only one pair of entangled photons at a time and, second, it can readily be done as it needs only linear optics elements.

Currently, the most widely used reliable source of polarization-entangled photons is parametric downconversion in a nonlinear optical crystal 13 . Here we need two-photon states that are maximally entangled both in polarization and in path degrees of freedom. Figure 1 shows the setup [14, 15] for generating pairs of polarization and path entangled photons. A pump pulse passing through the crystal can create, with a small probability, entangled pairs of photons in the spatial (path) modes $d_{1}$ and $u_{2}$. For definiteness, we assume that the entangled photon pairs are in the polarization-entangled state $\left|\Psi^{-}\right\rangle_{12}=\frac{1}{\sqrt{2}}\left(|H\rangle_{1}|V\rangle_{2}-|V\rangle_{1}|H\rangle_{2}\right)$, where $|H\rangle$ $(|V\rangle)$ stands for photons with horizontal (vertical) polarization. Now if the pump is reflected through the crystal a second time, then there is another possibility for pro- 


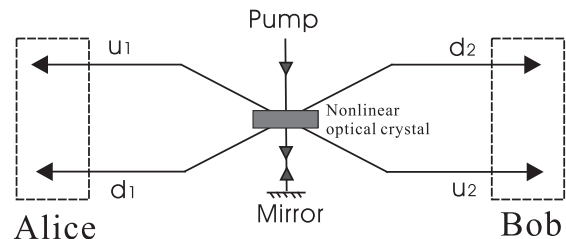

FIG. 1: Setup for generating pairs of photons entangled both in polarization and path.

ducing entangled pairs of photons into the path modes $u_{1}$ and $d_{2}$ that are opposite to the first modes $d_{1}$ and $u_{2}$. The two possible ways of producing the entangled photon pairs may interfere 14]. By properly adjusting the distance between the mirror and the crystal, the setup in Fig. 1 generates the doubly entangled two-photon state 15

$$
|\Psi\rangle_{12}=\frac{1}{2}\left(|H\rangle_{1}|V\rangle_{2}-|V\rangle_{1}|H\rangle_{2}\right)\left(|u\rangle_{1}|d\rangle_{2}-|d\rangle_{1}|u\rangle_{2}\right),
$$

which is just the desired state entangled maximally both in polarization and in path. Here photon- 1 and photon- 2 are, respectively, possessed by two observers, Alice and Bob, who are space-like separated; $|u\rangle$ and $|d\rangle$ denote two orthonormal path states of photons. With emphasis, we note that the state (11) indeed corresponds to the case where there is one and only one pair production after the pump pulse passes through the BBO crystal twice.

One can define the following Pauli-type operators for both the polarization and the path degrees of freedom:

$$
\begin{aligned}
& \sigma_{x}=|H\rangle\langle V|+| V\rangle\left\langle H\left|, \quad \sigma_{z}=\right| H\right\rangle\langle H|-| V\rangle\langle V| ; \\
& \sigma_{x}^{\prime}=|u\rangle\langle d|+| d\rangle\left\langle u\left|, \quad \sigma_{z}^{\prime}=\right| u\right\rangle\langle u|-| d\rangle\langle d| .
\end{aligned}
$$

For convenience and clarity, in the following we denote $z_{i}=\sigma_{z i}, x_{i}=\sigma_{x i}, z_{i}^{\prime}=\sigma_{z i}^{\prime}, x_{i}^{\prime}=\sigma_{x i}^{\prime}(i=1,2)$ and, use $(\cdot)$ to separate operators or operator products that can be identified as EPR's local "elements of reality". Then one can easily check the following eigenequations

$$
\begin{aligned}
& z_{1} \cdot z_{2}|\Psi\rangle_{12}=-|\Psi\rangle_{12}, z_{1}^{\prime} \cdot z_{2}^{\prime}|\Psi\rangle_{12}=-|\Psi\rangle_{12} \\
& x_{1} \cdot x_{2}|\Psi\rangle_{12}=-|\Psi\rangle_{12}, x_{1}^{\prime} \cdot x_{2}^{\prime}|\Psi\rangle_{12}=-|\Psi\rangle_{12} \\
& z_{1} z_{1}^{\prime} \cdot z_{2} \cdot z_{2}^{\prime}|\Psi\rangle_{12}=|\Psi\rangle_{12} \\
& x_{1} x_{1}^{\prime} \cdot x_{2} \cdot x_{2}^{\prime}|\Psi\rangle_{12}=|\Psi\rangle_{12} \\
& z_{1} \cdot x_{1}^{\prime} \cdot z_{2} x_{2}^{\prime}|\Psi\rangle_{12}=|\Psi\rangle_{12} \\
& x_{1} \cdot z_{1}^{\prime} \cdot x_{2} z_{2}^{\prime}|\Psi\rangle_{12}=|\Psi\rangle_{12} \\
& z_{1} z_{1}^{\prime} \cdot x_{1} x_{1}^{\prime} \cdot z_{2} x_{2}^{\prime} \cdot x_{2} z_{2}^{\prime}|\Psi\rangle_{12}=-|\Psi\rangle_{12}
\end{aligned}
$$

Equations (3)-(9) contain only local operators, i.e., $\left(z_{1}\right.$, $z_{1}^{\prime}, x_{1}, x_{1}^{\prime}, z_{1} z_{1}^{\prime}, x_{1} x_{1}^{\prime}, z_{1} \cdot x_{1}^{\prime}, x_{1} \cdot z_{1}^{\prime}$, and $\left.z_{1} z_{1}^{\prime} \cdot x_{1} x_{1}^{\prime}\right)$ for Alice and $\left(z_{2}, z_{2}^{\prime}, x_{2}, x_{2}^{\prime}, z_{2} \cdot z_{2}^{\prime}, x_{2} \cdot x_{2}^{\prime}, z_{2} x_{2}^{\prime}, x_{2} z_{2}^{\prime}\right.$, and $\left.z_{2} x_{2}^{\prime} \cdot x_{2} z_{2}^{\prime}\right)$ for Bob. In particular, Eqs. (3)-(9) allow Alice (Bob) to assign values with certainty to Bob's local operators $z_{2}, z_{2}^{\prime}, x_{2}, x_{2}^{\prime}, z_{2} x_{2}^{\prime}$, and $x_{2} z_{2}^{\prime}$ (Alice's local operators $z_{1}, z_{1}^{\prime}, x_{1}, x_{1}^{\prime}, z_{1} z_{1}^{\prime}$, and $\left.x_{1} x_{1}^{\prime}\right)$ by measuring her local observables (his local observables) without in any way disturbing Bob's (Alice's) photon. It is the idea of EPR's criterion of elements of reality to establish a local realistic interpretation of the quantum-mechanical results (3)-(9) by assuming that the individual value of any operator $\left(z_{1}, z_{1}^{\prime}, x_{1}, x_{1}^{\prime}, z_{1} z_{1}^{\prime}\right.$, and $\left.x_{1} x_{1}^{\prime}\right)$ at Alice's side and $\left(z_{2}, z_{2}^{\prime}, x_{2}, x_{2}^{\prime}, z_{2} x_{2}^{\prime}\right.$, and $\left.x_{2} z_{2}^{\prime}\right)$ at Bob's side is predetermined. These predetermined values are denoted by $v\left(z_{i}\right), v\left(z_{i}^{\prime}\right), v\left(x_{i}\right), v\left(x_{i}^{\prime}\right), v\left(z_{1} z_{1}^{\prime}\right), v\left(x_{1} x_{1}^{\prime}\right), v\left(z_{2} x_{2}^{\prime}\right)$, and $v\left(x_{2} z_{2}^{\prime}\right)$ with $v= \pm 1$. To be consistent with Eqs. (3)-(9), local realistic theories thus predict

$$
\begin{aligned}
& v\left(z_{1}\right) v\left(z_{2}\right)=-1, \quad v\left(z_{1}^{\prime}\right) v\left(z_{2}^{\prime}\right)=-1 \\
& v\left(x_{1}\right) v\left(x_{2}\right)=-1, \quad v\left(x_{1}^{\prime}\right) v\left(x_{2}^{\prime}\right)=-1 \\
& v\left(z_{1} z_{1}^{\prime}\right) v\left(z_{2}\right) v\left(z_{2}^{\prime}\right)=1, v\left(x_{1} x_{1}^{\prime}\right) v\left(x_{2}\right) v\left(x_{2}^{\prime}\right)=1 \\
& v\left(z_{1}\right) v\left(x_{1}^{\prime}\right) v\left(z_{2} x_{2}^{\prime}\right)=1, v\left(x_{1}\right) v\left(z_{1}^{\prime}\right) v\left(x_{2} z_{2}^{\prime}\right)=1 \\
& v\left(z_{1} z_{1}^{\prime}\right) v\left(x_{1} x_{1}^{\prime}\right) v\left(z_{2} x_{2}^{\prime}\right) v\left(x_{2} z_{2}^{\prime}\right)=-1
\end{aligned}
$$

But in fact, Eqs. (10)-(14) are mutually inconsistent: Multiplying Eqs. (10)-(13), one gets $v\left(z_{1} z_{1}^{\prime}\right) v\left(x_{1} x_{1}^{\prime}\right) v\left(z_{2} x_{2}^{\prime}\right) v\left(x_{2} z_{2}^{\prime}\right)=1$ due to the fact that $v^{2}\left(z_{i}\right)=v^{2}\left(z_{i}^{\prime}\right)=v^{2}\left(x_{i}\right)=v^{2}\left(x_{i}^{\prime}\right)=1$, and this is then in conflict with Eq. (14). Thus, the quantum-mechanical predictions (3)-(91) are incompatible with the ones imposed by local realistic theories. The contradiction between QM and local realism occurs for definite predictions and for all (100\%) of the photon pairs. This completes the demonstration of an all-versus-nothing nonlocality for our two-photon case.

An important point deserves further comment. It is well known that the original GHZ argument needs at least three spatially separated particles in order to establish the properties used in the argument as EPR's elements of reality. Therefore the question arises whether it is also possible to achieve the same in a two-particle situation as suggested in this paper. We are able to achieve this goal for two reasons: First, the number of variables used in the argument is enlarged compared to the original GHZ argument. Second, and most importantly, the nine variables can be arranged in three groups of three each, where the three variables of each group are measured by one and the same apparatus when establishing them as ERP's elements of reality, as we will show below. This eliminates the necessity of an argument based on counterfactuality as it is not necessary to assume any of these variables to be independent of experimental context.

Actually, the above argument can be understood from another perspective. By defining $|H\rangle|u\rangle \equiv|0\rangle,|H\rangle|d\rangle \equiv$ $|1\rangle,|V\rangle|u\rangle \equiv|2\rangle$ and $|V\rangle|d\rangle \equiv|3\rangle,|\Psi\rangle_{12}$ can be rewritten as $|\Psi\rangle_{12}=\frac{1}{2}\left(|0\rangle_{1}|3\rangle_{2}-|1\rangle_{1}|2\rangle_{2}-|2\rangle_{1}|1\rangle_{2}+|3\rangle_{1}|0\rangle_{2}\right)$, which is, in fact, a two-particle maximally-entangled state in a four-dimensional Hilbert space. This then im- 
plies that the GHZ-type argument has been indeed generalized to the case with only two entangled four-level particles. In contrast to the original GHZ proposal, our scheme requires only two space-like separated regions.

In a real experiment, the perfect correlations and ideal measurement devices are practically impossible. To face this difficulty, a Bell-Mermin inequality for $|\Psi\rangle_{12}$ is desirable. Similarly to Ref. [1], one can introduce the operator $\mathcal{O}=-z_{1} \cdot z_{2}-z_{1}^{\prime} \cdot z_{2}^{\prime}-x_{1} \cdot x_{2}-x_{1}^{\prime} \cdot x_{2}^{\prime}+z_{1} z_{1}^{\prime} \cdot z_{2} \cdot z_{2}^{\prime}+x_{1} x_{1}^{\prime}$. $x_{2} \cdot x_{2}^{\prime}+z_{1} \cdot x_{1}^{\prime} \cdot z_{2} x_{2}^{\prime}+x_{1} \cdot z_{1}^{\prime} \cdot x_{2} z_{2}^{\prime}-z_{1} z_{1}^{\prime} \cdot x_{1} x_{1}^{\prime} \cdot z_{2} x_{2}^{\prime} \cdot x_{2} z_{2}^{\prime}$. It can be directly seen from Eqs. (3)-(9) that $\mathcal{O}$ satisfies

$$
\mathcal{O}|\Psi\rangle_{12}=9|\Psi\rangle_{12}
$$

However, following Ref. 11] local realistic theories predict the observed values of $\mathcal{O}$

$$
\langle\mathcal{O}\rangle_{L R T} \leq 7,
$$

which is in contradiction with the quantum mechanical prediction (15). For observing the violation of the inequality (16), one needs the doubly-entangled state with a visibility better than $7 / 9 \approx 77.8 \%$. Here, we would like to mention that BI for "qudits" have more resistance to noise and the required visibility can be reduced to about $69 \%$ for four-dimensional systems $[8,9]$.

Though the above argument is formally similar to the reasoning of Cabello's theorem 10, 11], at this stage the advantages of our scheme are already manifest. Our argument works for two entangled photons, whose path and polarization degrees of freedom are used. Experimentally, manipulating a single pair of entangled photons is much easier than manipulating two pairs. These features are essential for an experimental test of the GHZ-type theorem proposed here.

We now further discuss the noncontextuality issue to validate our all-versus-nothing quantum nonlocality argument. In the argument the same operators may appear in different equations (3)-(9). For example, $z_{1} z_{1}^{\prime}$ and $x_{1} x_{1}^{\prime}$ not only appear separately in Eqs. (5) and (6), but also appear jointly in Eq. (9). In order for the argument to hold, it is, however, necessary to assign always a single value to the same operator, though it can appear in different equations. Therefore, one either has to assume noncontexuality (e.g., measurement of $z_{1} z_{1}^{\prime}$ does not disturb the value of $x_{1} x_{1}^{\prime}$ and vice versa) or one has to be able to measure $z_{1} z_{1}^{\prime}, x_{1} x_{1}^{\prime}$ and $z_{1} z_{1}^{\prime} \cdot x_{1} x_{1}^{\prime}$ with the same apparatuses. Lvovsky noticed that this would then require quantum controlled-NOT (CNOT) operation to apply on all $(100 \%)$ photon pairs to demonstrate Cabello's quantum nonlocality in the original proposal with two photon pairs. Unfortunately, this requires nonlinear optics.

This CNOT operation is equivalent to making a complete Bell-state discrimination (see Refs. 10, 11, 12]), in which the Bell states are $\left|\Psi^{ \pm}\right\rangle=\frac{1}{\sqrt{2}}(|H\rangle|V\rangle \pm|V\rangle|H\rangle)$ and $\left|\Phi^{ \pm}\right\rangle=\frac{1}{\sqrt{2}}(|H\rangle|H\rangle \pm|V\rangle|V\rangle)$. It has been well known initially in the context of quantum teleportation [16, 17, 18] that such a full Bell-state measurement is impossible with only linear optics and necessitates nonlinear optical interactions at a single-photon level [19], which is very challenging experimentally. Thus, an experimental test of Cabello's nonlocality cannot be achieved by existing technology 12 .

However, within our two-photon proposal, the above problem does not exist since quantum CNOT operations can be easily implemented between two different degrees of freedom of single photons. Actually, measuring $z_{1} z_{1}^{\prime}$. $x_{1} x_{1}^{\prime}$ in the present scheme is equivalent to performing a complete Bell-state measurement, with the four Bell states

$$
\begin{aligned}
& \left|\psi^{ \pm}\right\rangle=\frac{1}{\sqrt{2}}(|H\rangle|d\rangle \pm|V\rangle|u\rangle), \\
& \left|\phi^{ \pm}\right\rangle=\frac{1}{\sqrt{2}}(|H\rangle|u\rangle \pm|V\rangle|d\rangle),
\end{aligned}
$$

instead of $\left|\Psi^{ \pm}\right\rangle$and $\left|\Phi^{ \pm}\right\rangle$. The complete discrimination of the four Bell states in Eq. (17) has been realized in the "two-particle analog" of the quantum teleportation experiment performed in Rome [18]. Such a complete Bell-state discrimination can be accomplished with linear optics and almost $100 \%$ efficiency.

Thus the difficulty of measuring simultaneously $z_{1} z_{1}^{\prime}$, $x_{1} x_{1}^{\prime}$ and $z_{1} z_{1}^{\prime} \cdot x_{1} x_{1}^{\prime}$ has been eliminated by our twophoton proposal. We now consider the question of how to measure the quantities such as $z_{1}, x_{1}^{\prime}$ and $z_{1} \cdot x_{1}^{\prime}$ in our two-photon scheme. It is obvious that the measurements of $z_{1}, x_{1}^{\prime}$ and $z_{1} \cdot x_{1}^{\prime}$ have to be performed on the single photon possessed by Alice. Therefore, in order to avoid the noncontexuality assumption, one must design an apparatus such that it can give the measurement results of $z_{1}, x_{1}^{\prime}$ and $z_{1} \cdot x_{1}^{\prime}$ simultaneously.

Similar considerations would thus lead to the following six apparatuses (similar apparatuses have been proposed by Simon et al. 21] in a different context), which are sufficient to solve the problem just mentioned. Apparatus-1 measures $z_{1}, x_{1}^{\prime}$ and $z_{1} \cdot x_{1}^{\prime}$; apparatus- 2 measures $x_{2}$, $x_{2}^{\prime}$ and $x_{2} \cdot x_{2}^{\prime}$; apparatus- 3 measures $z_{1}^{\prime}, x_{1}$ and $x_{1} \cdot z_{1}^{\prime}$; apparatus- 4 measures $z_{2}, z_{2}^{\prime}$ and $z_{2} \cdot z_{2}^{\prime}$; apparatus-5 measures $z_{1} z_{1}^{\prime}, x_{1} x_{1}^{\prime}$ and $z_{1} z_{1}^{\prime} \cdot x_{1} x_{1}^{\prime}$; apparatus- 6 measures $z_{2} x_{2}^{\prime}, x_{2} z_{2}^{\prime}$ and $z_{2} x_{2}^{\prime} \cdot x_{2} z_{2}^{\prime}$. Fortunately enough, each of these apparatuses measures different local observables and, more importantly, the six apparatuses can be realized without any mutual conflict.

Figures 2 (a-d) show the first four apparatuses, which require only simple linear optical elements [e.g., the beam splitters (BS), polarizing beam splitters (PBS) and half wave plates (HWP) rotated at $45^{\circ}$ ] and singlephoton detectors. Note that a PBS reflects $V$ photons and transmits $H$ photons, and a BS (HWP) affects the following transformations: $|u\rangle \rightarrow(|u\rangle+|d\rangle) / \sqrt{2}$ and $|d\rangle \rightarrow(|u\rangle-|d\rangle) / \sqrt{2}(|H\rangle \rightarrow(|H\rangle+|V\rangle) / \sqrt{2}$ and 

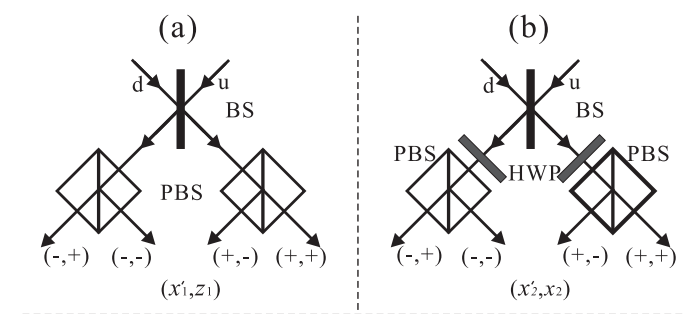

(c)

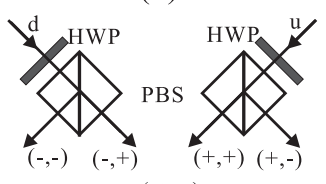

$\left(z_{1}^{1}, x_{1}\right)$

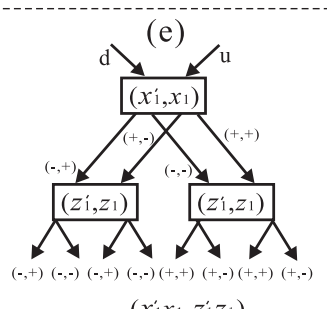

$\left(x_{1}^{\prime} x_{1}, z_{1}^{\prime} z_{1}\right)$

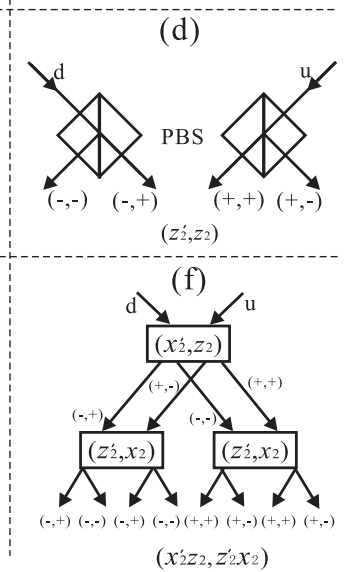

$\left(x_{2}^{\prime} z_{2}, z_{2}^{\prime} x_{2}\right)$
FIG. 2: Six apparatuses for measuring $z_{1}, x_{1}^{\prime}$ and $z_{1} \cdot x_{1}^{\prime}$ (a); $x_{2}, x_{2}^{\prime}$ and $x_{2} \cdot x_{2}^{\prime}(\mathrm{b}) ; z_{1}^{\prime}, x_{1}$ and $x_{1} \cdot z_{1}^{\prime}(\mathrm{c}) ; z_{2}, z_{2}^{\prime}$ and $z_{2} \cdot z_{2}^{\prime}$ (d); $z_{1} z_{1}^{\prime}, x_{1} x_{1}^{\prime}$ and $z_{1} z_{1}^{\prime} \cdot x_{1} x_{1}^{\prime}(\mathrm{e}) ; z_{2} x_{2}^{\prime}, x_{2} z_{2}^{\prime}$ and $z_{2} x_{2}^{\prime} \cdot x_{2} z_{2}^{\prime}$ (f). By \pm , we mean \pm 1 .

$|V\rangle \rightarrow(|H\rangle-|V\rangle) / \sqrt{2})$. Let us consider, e.g., apparatus 1. Since apparatus 1 measures $z_{1}$ and $x_{1}^{\prime}$ simultaneously, it actually also gives the measurement result of $z_{1} \cdot x_{1}^{\prime}$. Figures 2(e) and 2(f) show the last two apparatuses, each of which is made up of three of the first four apparatuses. Figure 2(e) [2(f)] also shows that the information obtained in the first stage of the measurement about the values of $x_{1}$ and $x_{1}^{\prime}\left(z_{2}\right.$ and $\left.x_{2}^{\prime}\right)$ must be partially erased in such a way that only information about the product $x_{1} x_{1}^{\prime}\left(z_{2} x_{2}^{\prime}\right)$ is retained, to enable the measurement of $z_{1} z_{1}^{\prime}\left(x_{2} z_{2}^{\prime}\right)$ at the last stage [21]. Apparatus 5 (apparatus 6) measures $z_{1} z_{1}^{\prime}$ and $x_{1} x_{1}^{\prime}\left(z_{2} x_{2}^{\prime}\right.$ and $\left.x_{2} z_{2}^{\prime}\right)$ simultaneously, and thus gives also the readout of $z_{1} z_{1}^{\prime} \cdot x_{1} x_{1}^{\prime}$ $\left(z_{2} x_{2}^{\prime} \cdot x_{2} z_{2}^{\prime}\right)$. It is worthwhile to note that apparatus 2(e) can be replaced with the apparatuses discriminating the four Bell states in Eq. (17). For apparatus 2(f), the situation is similar.

To summarize, we have demonstrated an all-versusnothing nonlocality for two photons, which are maximally entangled both in polarization and in path degrees of freedom. Since the required measurement of local operators can be implemented with linear optics, our twophoton proposal is well within the reach of current quantum optical technology. Note that, untill now, there is only one experiment [5] performed to test the GHZ nonlocality, a kind of all-versus-nothing nonlocality. In this respect, the feasible experimental scheme, as we suggested in this work, is highly desirable.
Finally, it is interesting to point out that using a single photon as a two-qubit (polarization and path qubits) system may find important applications in other contexts, e.g., testing the Kochen-Specker theorem 20] with single particles 21, 22], quantum computing 23], quantum cryptography [24], and entanglement purification [15]. A recent study shows that the path-polarization entangled two-photon states (11) can also be used to implement deterministic and efficient quantum cryptography [25].

We thank Jiang-Feng Du, Zhi Zhao and Marek Zukowski for useful discussions. This work was supported by the National Natural Science Foundation of China, the Chinese Academy of Sciences and the National Fundamental Research Program (under Grant No. 2001CB309300). This work was also supported by the Austrian Science Foundation FWF, Project No. F1506.

[1] J.S. Bell, Physics (Long Island City, N.Y.) 1, 195 (1964).

[2] A. Einstein, B. Podolsky, and N. Rosen, Phys. Rev. 47, 777 (1935).

[3] D.M. Greenberger, M.A. Horne, and A. Zeilinger, in Bell's Theorem, Quantum Theory, and Conceptions of the Universe, edited by M. Kafatos (Kluwer Academic, Dordrecht, 1989).

[4] D.M. Greenberger, M.A. Horne, A. Shimony, and A. Zeilinger, Am. J. Phys. 58, 1131 (1990).

[5] J.-W. Pan et al., Nature (London) 403, 515 (2000).

[6] L. Hardy, Phys. Rev. Lett. 71, 1665 (1993).

[7] N.D. Mermin, Ann. N.Y. Acad. Sci. 755, 616 (1995).

[8] D. Kaszlikowski et al., Phys. Rev. Lett. 85, 4418 (2000).

[9] T. Durt et al., Phys. Rev. A 64, 024101 (2001); J.-L. Chen et al., ibid. 64, 052109 (2001); D. Collins et al., Phys. Rev. Lett. 88, 040404 (2002).

[10] A. Cabello, Phys. Rev. Lett. 86, 1911 (2001).

[11] A. Cabello, Phys. Rev. Lett. 87, 010403 (2001).

[12] A.I. Lvovsky, Phys. Rev. Lett. 88, 098901 (2002).

[13] P.G. Kwiat et al., Phys. Rev. Lett. 75, 4337 (1995).

[14] T.J. Herzog, J.G. Rarity, H. Weinfurter, and A. Zeilinger, Phys. Rev. Lett. 72, 629 (1994).

[15] C. Simon and J.-W. Pan, Phys. Rev. Lett. 89, 257901 (2002).

[16] C.H. Bennett et al., Phys. Rev. Lett. 70, 1895 (1993).

[17] D. Bouwmeester et al., Nature (London) 390, 575 (1997); J.-W. Pan et al., Phys. Rev. Lett. 80, 3891 (1998).

[18] D. Boschi et al., Phys. Rev. Lett. 80, 1121 (1998).

[19] L. Vaidman and N. Yoran, Phys. Rev. A 59, 116 (1999); N. Lütkenhaus et al., ibid. 59, 3295 (1999).

[20] S. Kochen and E. P. Specker, J. Math. Phys. 17, 59 (1967).

[21] C. Simon, M. Żukowski, H. Weinfurter, and A. Zeilinger, Phys. Rev. Lett. 85, 1783 (2000).

[22] M. Michler, H. Weinfurter, and M. Żukowski, Phys. Rev. Lett. 84, 5457 (2000).

[23] B.-G. Englert et al., Phys. Rev. A 63, 032303 (2001).

[24] A. Beige et al., J. Phys. A: Math. Gen. 35, L407 (2002).

[25] Z. Zhao et al., quant-ph/0211098 